\title{
Region-based Comer Detection by Radial Projection
}

\author{
Daeho Lee*, SeungGwan Lee, and Jin Hyuk Choi \\ Humanitas College, Kyung Hee University, Yongin 446-701, Korea
}

(Received February 9, 2011 : accepted April 11, 2011)

\begin{abstract}
We propose a novel method which detects convex and concave corners using radial projection. The sum of two neighbors' differences at the local maxima or minima of the radial projection is compared with the angle threshold for detecting corners. In addition, the use of oriented bounding box trees and partial circles makes it possible to detect the corners of complex shapes. The experimental results show that the proposed method can separately detect the convex and concave corners, and that this method is scale invariant.
\end{abstract}

Keywords: Radial projection, Corner detection, Shape analysis, Feature extraction

OCIS codes : (100.5010) Pattern recognition; (330.1880) Detection

\section{INTRODUCTION}

Pattern recognition is widely used for various applications in the fields of medicine, industry, meteorology, and more [1-3]. Shape analysis methods play an important role in pattern recognition, matching, registration, and analysis [4]. Shape analysis can be classified into two methods: boundary based or internal (i.e., region) based approaches. The boundary based methods generally use the scale-space filters $[5,6]$ or Fourier transforms $[7,8]$ of the boundary, while the internal based methods use the moment [5, 9] and projection [10-12] of the object regions.

The methods which use the Fourier transforms and moments are used to classify the type of object, while the methods which use the scale-space filters and projections are used to find the dominant points and boundary locations. The shapes which can be located by the projections are flat or rectangular boundaries because those functions are generally based on the Cartesian coordinates. However, the projection functions based on polar coordinates may be used for other applications. The ring projection function, which is defined as the accumulated values of the image along a specific radius, can be used as a rotation-invariant feature [11]. The radial projection function, which is defined as the accumulated values of the image at a specific angle, can be used as a feature for topographical shapes [12].

In this paper, we propose a novel method which can separately detect convex and concave corners by using radial projection. This method is scale invariant.

\section{RADIAL PROJECTION FOR CORNER DETECTION}

The radial projection function based on polar coordinates is defined, as follows:

$$
p_{\text {rad }}(\theta)=\sum_{k} I\left(r_{k} \cos \theta, r_{k} \sin \theta\right),
$$

where $P_{\operatorname{rad}}(\theta)$ is the radially accumulated value at a specific angle, $\theta$. To calculate the radial projection function, the origin of the shape is first estimated to be the centroid of the shape. The radial projection is the function of the angles, so that the function can be represented by the discrete angle variables, as follows:

$$
p_{\text {rad }}\left(\theta_{i}\right)=\left\{p_{\text {rad }}\left(\theta_{0}\right), p_{\text {rad }}\left(\theta_{1}\right), \cdots p_{r a d}\left(\theta_{N-1}\right)\right\},
$$

where $P_{\mathrm{rad}}\left(\theta_{i}\right)$ should be expressed within the range $[0,2 \pi)$, so that the difference between the angles is

$$
\left|\theta_{i}-\theta_{i-1}\right|=\frac{2 \pi}{N}
$$

\footnotetext{
*Corresponding author: nize@khu.ac.kr

Color versions of one or more of the figures in this paper are available online.
} 


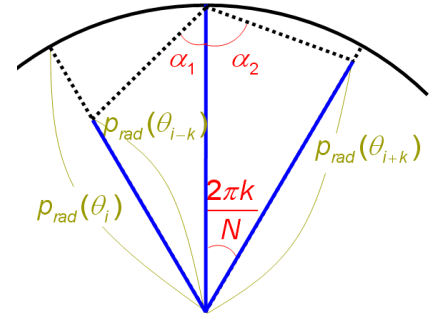

convex

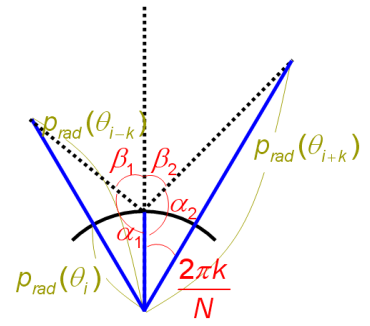

concave
FIG. 1. Radial projection for corner detection.

To detect the corners from the computed $P_{\operatorname{rad}}\left(\theta_{i}\right)$, the local maximum and minimum positions are assigned as the convex and concave corner directions, respectively, and then the corner angles are computed by the sum of the two neighbor differences $\left(\alpha_{1}\right.$ and $\left.\alpha_{2}\right)$, as shown in Fig. 1, where the one angle is calculated by

$$
\alpha_{1}=\cos ^{-1}\left(\frac{-p_{\text {rad }}\left(\theta_{i-k}\right)^{2}+p_{\text {rad }}\left(\theta_{i}\right)^{2}+c^{2}}{2 p_{\text {rad }}\left(\theta_{i}\right) c}\right),
$$

where $c$ is

$$
\sqrt{p_{\text {rad }}\left(\theta_{i}\right)^{2}+p_{\text {rad }}\left(\theta_{i-k}\right)^{2}-2 p_{\text {rad }}\left(\theta_{i-k}\right) p_{\text {rad }}\left(\theta_{i}\right) \cos \left(\frac{2 \pi k}{N}\right)},
$$

and where $k$ is the neighborhood angle size for detecting corner angles.

As shown in Fig. 1, if the corner angle $\left(\alpha_{1}+\alpha_{2}\right)$ of $\theta_{i}$ is concave, then it is less than $\pi$. Otherwise, the corner angle $\left(\beta_{1}+\beta_{2}\right)$ is concave, and it equals $2 \pi-\left(\alpha_{1}+\alpha_{2}\right)$.

\section{RADIAL PROJECTION OF COMPLEX SHAPES}

It is impossible to analyze a complex shape with only one origin. Thus, we separate the shape into partial regions using tight-fitting oriented bounding box trees (OBB Trees) [13]. The partial shapes then become slab-sided shapes bounded by rectangles, and the corners of each shape are detected using the elliptic model.

Ellipses each have a major axis and a minor axis. The direction of the major axis of the approximated ellipse is calculated as the average angle from the centroid by

$$
2 \phi=\sum_{(x, y) \in S} \tan ^{-1}\left(\frac{\sin \left(2 \tan ^{-1}\left(\frac{y-c_{y}}{x-c_{x}}\right)\right)}{\cos \left(2 \tan ^{-1}\left(\frac{y-c_{y}}{x-c_{x}}\right)\right)}\right),
$$

where $\left(c_{x}, c_{y}\right)$ denotes the original origin, $S$ denotes a

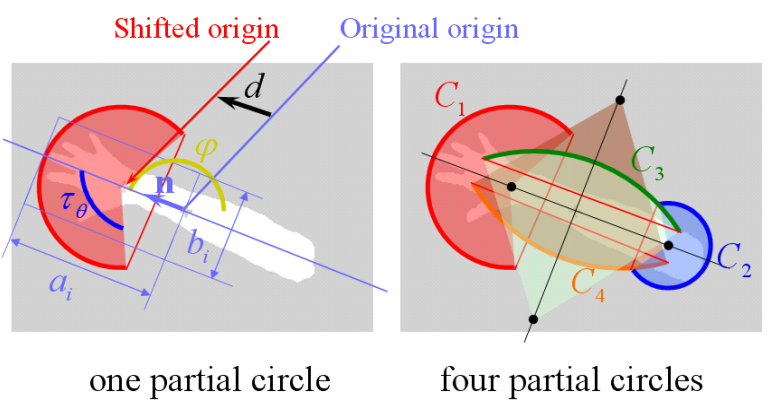

FIG. 2. Radial projection of slab-sided shapes.

slab-sided shape, and $-\pi / 2 \pi \leq \phi<\pi / 2$.

After detecting $\phi$, we shift the original origin in the direction of $\mathbf{n}=(\cos \phi, \sin \phi)$. The shifted length $(d)$ may be determined using the local axis lengths $\left(a_{i}\right.$ and $\left.b_{i}\right)$ of the ellipse, as follows:

$$
d=a_{i}-\kappa b_{i},
$$

where $a_{i}$ and $b_{i}$ denote the transverse diameter and the conjugate diameter of the half region with respect to the shifted direction, respectively, and $\kappa$ denotes a scaling factor for a partial circle. By using the shifted origin, the radial projection is calculated within a limited angle range $\left(\tau_{\theta}\right)$, as shown in Fig. 2, where $\tau_{\theta}$ may be determined by $d$.

The origin may be shifted in the directions parallel to and perpendicular to $\mathbf{n}$, so that four partial circles are generated, as shown in Fig. 2.

\section{EXPERIMENTAL RESULTS}

To evaluate the proposed method, we tested it using six images, as shown in Fig. 3. The test was performed for $N=180$ and $k=10$, and corners with angles less than $2 \pi / 3$ were detected. In Fig. 3, the origins are marked with red crosses, the convex corners are marked with large dots, the concave corners are marked with small dots.

For the six shapes tested, we calculate the MSE (mean square error), which is defined by

$$
\mathrm{MSE}=\frac{1}{n} \sum_{i=1}^{n}\left(\hat{\varphi}_{i}-\varphi_{i}\right)^{2}
$$

where $\hat{\varphi_{1}}$ and $\varphi_{1}$ are the calculated angle and the ground truth angle of the i-th corner (in radians), respectively, and $n$ is the number of corners detected. The MSEs of the six shapes tested are very low, as shown in Table 1 .

To evaluate the performance with regard to the scale invariant measure, we tested the images with a zoom of $2 \mathrm{x}$ and $4 \mathrm{x}$ with the same parameters $(N$ and $k)$ and then calculated the standard deviations of the detected corners. The average of the standard deviation $(\bar{\sigma})$ of the tested 


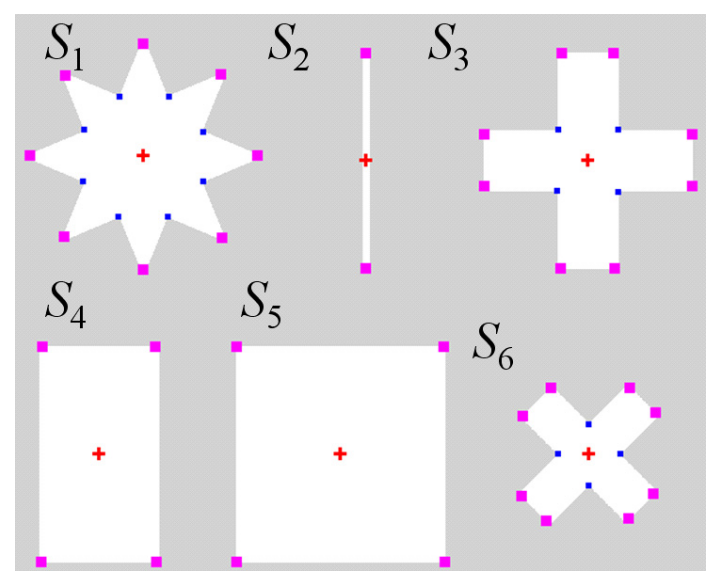

FIG. 3. Corner detection results.

TABLE 1. MSE of the tested six shapes

\begin{tabular}{c|c|c|c|c|c|c}
\hline \hline Shape & S1 & S2 & S3 & S4 & S5 & S6 \\
\hline MSE & 0.0061 & 0.0076 & 0.0041 & 0.0024 & 0.0004 & 0.0067 \\
\hline
\end{tabular}

TABLE 2. $\bar{\sigma}$ of the tested six shapes

\begin{tabular}{c|c|c|c|c|c|c}
\hline Shape & S1 & S2 & S3 & S4 & S5 & S6 \\
\hline MSE & 0.0118 & 0.0047 & 0.0043 & 0.0032 & 0.0059 & 0.0153 \\
\hline
\end{tabular}

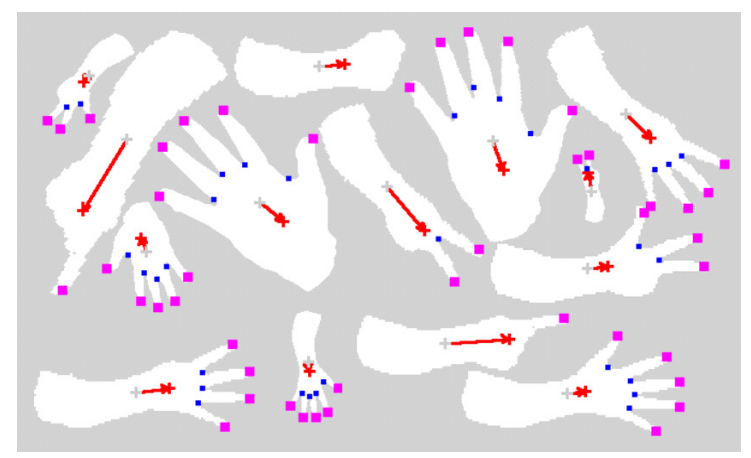

FIG. 4. Fingertip and finger valley detection results.

shapes is very low, as shown in Table 2. Previous methods, however, were very sensitive to scale parameters, so that the corners could not be reliably detected.

To evaluate the performance for complex shapes, we tested our method for fingertip detection. The original images were binarized using color differences. The partial circles were generated using $\kappa=1.7$, the radial projections were calculated for $N=180$ and $k=5$ within $\tau_{\theta}<3 \pi / 2$, and corners with angles less than $2 \pi / 3$ were detected. The results are shown in Fig. 4, where the bright (gray) crosses indicate the original origins, and the dark (red) crosses indicate the shifted origins. The proposed method detected the convex and concave corners separately, so that the fingers and the finger valleys were correctly detected.

\section{CONCLUSIONS}

This paper proposes a novel method which detects convex and concave corners by using radial projection. The corner angle is calculated using the sum of two neighbor differences at the local maxima or minima of the radial projection function. The use of oriented bounding box trees and partial circles enables the detection of the corners of complex shapes. Moreover, this method can detect the same corners of scaled and rotated images with the same parameters. Therefore, the proposed method may play a strong role in corner detection, image matching, and other applications.

\section{REFERENCES}

1. M. Zhenjiang, "Zernike moment-based image shape analysis and its application," Patt. Recogn. Lett. 21, 169-177 (2000).

2. D. Lee and J. H. Choi, "Precise detection of car license plates by locating main characters," J. Opt. Soc. Korea 14, 376-382 (2010).

3. M. K. Kim, "Adaptive thresholding technique for binarization of license plate image," J. Opt. Soc. Korea 14, 368-375 (2010).

4. S. Loncaric, "A survey of shape analysis techniques," Patt. Recogn. 31, 983-1001 (1998).

5. S. Pei and C. Lin, "The detection of dominant points on digital curves by scale-space filtering," Patt. Recogn. 25, 1307-1314 (1992).

6. C. Teh and R. T. Chin, "On the detection of dominant points on digital curves," IEEE Trans. Patt. Anal. Mach. Intell. 11, 859-872 (1989).

7. C. T. Zahn and R. Z. Roskies, "Fourier descriptors for plane closed curves," IEEE Trans. Comp. 21, 269-281 (1972).

8. G. H. Granlund, "Fourier preprocessing for hand print character recognition," IEEE Trans. Comp. 21, 195-201 (1972).

9. M. Hu, "Visual pattern recognition by moment invariants," IRE Trans. Inf. Theory 8, 179-187 (1962).

10. Z. Zhou and X. Geng, "Projection functions for eye detection," Patt. Recogn. 37, 1049-1056 (2004).

11. D. Tsai and Y. Tsai, "Rotation-invariant pattern matching with color ring-projection," Patt. Recogn. 35, 131-141 (2002).

12. Y. Kwon, H. Park, J. Liu, and M. A. Nascimento, "Radial projection: a feature extraction method for topographical shapes," LNCS 3769, $582-593$ (2005).

13. S. Gottschalk, M. C. Lin, and D. Manocha, "OBB tree: a hierarchical structure for rapid interference detection," in Proc. Int. Conf. on Computer Graphics and Interactive Techniques (New Orleans, USA, Aug. 1996), pp. 171-180. 